\title{
DIVIDEND MOMENTS IN THE DUAL RISK MODEL: EXACT AND APPROXIMATE APPROACHES
}

\author{
BY
}

\author{
Eric C.K. Cheung and Steve Drekic
}

\begin{abstract}
In the classical compound Poisson risk model, it is assumed that a company (typically an insurance company) receives premium at a constant rate and pays incurred claims until ruin occurs. In contrast, for certain companies (typically those focusing on invention), it might be more appropriate to assume expenses are paid at a fixed rate and occasional random income is earned. In such cases, the surplus process of the company can be modelled as a dual of the classical compound Poisson model, as described in Avanzi et al. (2007). Assuming further that a barrier strategy is applied to such a model (i.e., any overshoot beyond a fixed level caused by an upward jump is paid out as a dividend until ruin occurs), we are able to derive integro-differential equations for the moments of the total discounted dividends as well as the Laplace transform of the time of ruin. These integro-differential equations can be solved explicitly assuming the jump size distribution has a rational Laplace transform. We also propose a discrete-time analogue of the continuous-time dual model and show that the corresponding quantities can be solved for explicitly leaving the discrete jump size distribution arbitrary. While the discrete-time model can be considered as a stand-alone model, it can also serve as an approximation to the continuous-time model. Finally, we consider a generalization of the so-called Dickson-Waters modification in optimal dividends problems by maximizing the difference between the expected value of discounted dividends and the present value of a fixed penalty applied at the time of ruin.
\end{abstract}

\section{KEYWORDS}

Dual model, barrier strategy, dividend moments, time of ruin, rational Laplace transform.

\section{INTRODUCTION}

In the continuous-time dual risk model, the company's surplus process $\{U(t)$, $t \geq 0\}$ with initial surplus $u=U(0)$ is given by 


$$
U(t)=u-c t+\sum_{i=1}^{N(t)} Y_{i}, t \geq 0,
$$

where $c$ is the constant rate of expenses per unit time, $\{N(t), t \geq 0\}$ is a Poisson process with rate $\lambda$, and $\left\{Y_{i}, i \in \mathbb{Z}^{+}\right\}$is a sequence of independent and identically distributed (i.i.d.) positive continuous random variables with finite mean, independent of $\{N(t), t \geq 0\}$. For convenience, we assume all $Y_{i}^{\prime}$ s have the same distribution as a generic random variable $Y$ which has probability density function (p.d.f.) $p($.$) , cumulative distribution function (c.d.f.) P($.), survival function (s.f.) $\bar{P}($.), and Laplace transform (of $p().) \tilde{p}($.$) . Under the model$ (1.1), the expected increase in surplus per unit time is $\mu=\lambda E(Y)-c$, and it is assumed that $\mu>0$, or equivalently, $E(Y)=c(1+\theta) / \lambda$ for some $\theta>0$. For a detailed study of the model (1.1), see Seal (1969, p. 116).

Avanzi et al. (2007) propose that a model with dynamics described by (1.1) might be appropriate for businesses (e.g., brokerage firms) that sell mutual funds or insurance products having a front-end load, or perhaps for companies experiencing occasional gains which can be modelled by a compound Poisson process. Pharmaceutical or petroleum companies fall into this latter category, and we can think of each upward jump to be the net present value of future income as a result of an invention or discovery. In addition, the model (1.1) might also be applicable in a life insurance setting, such as a company which regularly pays annuities and which can (randomly) earn a portion of the reserves when, for example, a policyholder dies. For a more detailed discussion of applications, we refer interested readers to Seal (1969, p. 116), Mazza and Rullière (2004), Avanzi et al. (2007), and references therein.

Now we further assume a barrier strategy is applied to the model described by (1.1). This means that when the surplus is less than or equal to a fixed barrier $b>0$, the modified surplus process behaves exactly like the one without a barrier. Conversely, any overshoot beyond level $b$ caused by an upward jump is paid out immediately as a dividend. We assume no further dividends are paid beyond the time of ruin, where ruin is said to occur if the surplus ever drops to 0. A typical sample path of the surplus process under such a barrier strategy is depicted in Figure 1 of Avanzi et al. (2007).

Under the above description of the dual model with dividend payments, we are mainly interested in two random variables in this paper, namely, $D_{u, b}$ and $T_{u, b}$, which represent respectively the present value of total dividends paid until ruin under a force of interest $\delta>0$ and the time of ruin, both with initial surplus $u$ and dividend barrier $b$. In what follows, we denote the $n$-th moment of $D_{u, b}$ by

$$
V_{n}(u ; b)=E\left(D_{u, b}^{n}\right), n \in \mathbb{N},
$$

where $\mathbb{N}=\{0\} \cup \mathbb{Z}^{+}$. The 0 -th moment, $V_{0}(u ; b)$, is assumed to be 1 for any values of $u$ and $b$. We remark that dividend payments occur at discrete points in time, and this is in contrast to the classical risk model where dividend payments 
consist of continuous streams of payments resulting from premium income. Furthermore, the Laplace transform of $T_{u, b}$ is defined as

$$
\phi(u ; b)=E\left(e^{-\delta T_{u, b}}\right),
$$

where $\delta>0$ represents the Laplace transform argument here. Indeed, the above quantity also represents the present value of a dollar payable at the time of ruin under a force of interest $\delta$. We point out that this quantity plays a key role in our study of optimal dividends problems, as will be evident in Section 7 of the paper.

Central to the evaluation of $V_{n}(u ; b)$ is the $n$-th equilibrium distribution of the jump size distribution $p$ (.) (if it exists). For $n \in \mathbb{N}$, we denote the p.d.f., c.d.f., s.f., and Laplace transform (of the p.d.f.) of the $n$-th equilibrium distribution of the random variable $Y$ by $p_{n}(),. P_{n}(),. \bar{P}_{n}($.$) , and \tilde{p}_{n}($.$) respectively,$ with the usual convention that $p_{0}()=.p($.$) . Furthermore, we denote the generic$ random variable having p.d.f. $p_{n}($.$) by { }_{n} Y$.

The remainder of the paper is organized as follows. In Section 2, integrodifferential equations satisfied by $V_{n}(u ; b)$ and $\phi(u ; b)$ are derived. These integro-differential equations are then solved explicitly in Section 3 assuming that the jump size random variable $Y$ is distributed as a combination of exponentials, whereas in Section 4 more general solutions are given under the assumption that the Laplace transform $\tilde{p}($.$) is a rational function. In Section 5, a dis-$ crete-time analogue of the continuous-time dual model is proposed and studied. In particular, we show that the corresponding dividend moments and the Laplace transform of the time of ruin in the discrete-time model can be solved explicitly while leaving the discrete jump size distribution arbitrary. The discretetime model can also serve as an approximation to the continuous-time model, and this is the focus of Section 6. In Section 7, we consider a generalization of the so-called Dickson-Waters modification in optimal dividends problems by maximizing the difference between the expected value of discounted dividends and the present value of a fixed penalty applied at the time of ruin. Some results in Avanzi et al. (2007) are extended here. We conclude the paper with Section 8 demonstrating some interesting numerical results and findings.

\section{INTEGRO-DIFFERENTIAL EQUATIONS FOR $V_{n}(u ; b)$ AND $\phi(u ; b)$}

\subsection{Dividend Moments}

Since ruin occurs immediately for an initial surplus of zero, we have

$$
V_{n}(0 ; b)=0, n \in \mathbb{Z}^{+} .
$$

Furthermore, if $u>b$, an initial dividend of $u-b$ is immediately paid out, with any future dividend payments being modelled by the quantity $D_{b, b}$. Therefore, applying a binomial expansion, we arrive at 


$$
V_{n}(u ; b)=\sum_{j=0}^{n}\left(\begin{array}{l}
n \\
j
\end{array}\right)(u-b)^{n-j} V_{j}(b ; b), \quad u>b ; n \in \mathbb{Z}^{+} .
$$

For $0<u \leq b$, we consider all possible events over a small time interval $[0, h]$ and obtain

$$
\begin{aligned}
V_{n}(u ; b)= & (1-\lambda h) e^{-n \delta h} V_{n}(u-c h ; b)+\lambda h e^{-n \delta h} \int_{0}^{b-(u-c h)} V_{n}(u-c h+y ; b) p(y) d y \\
& +\lambda h e^{-n \delta h} \int_{b-(u-c h)}^{\infty} \sum_{j=0}^{n}\left(\begin{array}{c}
n \\
j
\end{array}\right)(u-c h+y-b)^{n-j} V_{j}(b ; b) p(y) d y+o(h) .
\end{aligned}
$$

Since $e^{-n \delta h}=1-n \delta h+o(h)$, the first term on the right-hand side can be expanded as $(1-\lambda h) e^{-n \delta h} V_{n}(u-c h ; b)=V_{n}(u ; b)-c h V_{n}^{\prime}(u ; b)-(\lambda+n \delta) h V_{n}(u ; b)+$ $o(h)$ via a Taylor series expansion of the quantity $V_{n}(u-c h ; b)$. Thus, cancelling $V_{n}(u ; b)$ on both sides of the above equation, dividing by $h$, and letting $h$ go to 0 , we get

$$
\begin{aligned}
& c V_{n}^{\prime}(u ; b)+(\lambda+n \delta) V_{n}(u ; b)-\lambda \int_{0}^{b-u} V_{n}(u+y ; b) p(y) d y \\
& \quad-\lambda \sum_{j=0}^{n}\left(\begin{array}{c}
n \\
j
\end{array}\right)\left(\int_{b-u}^{\infty}\{y-(b-u)\}^{n-j} p(y) d y\right) V_{j}(b ; b)=0, \quad 0<u \leq b ; n \in \mathbb{Z}^{+} .
\end{aligned}
$$

We remark that when $n=1,(2.3)$ reduces to equation (2.3) of Avanzi et al. (2007).

The right-hand side of (2.3) involves stop-loss moments. By the well-known link between stop-loss moments and equilibrium distributions (e.g., see Willmot et al. (2005, equation (1.5))), (2.3) can be re-expressed as

$$
\begin{aligned}
c V_{n}^{\prime}(u ; b) & +(\lambda+n \delta) V_{n}(u ; b)-\lambda \int_{u}^{b} V_{n}(y ; b) p(y-u) d y \\
& -\lambda \sum_{j=0}^{n}\left(\begin{array}{c}
n \\
j
\end{array}\right) \bar{P}_{n-j}(b-u) E\left(Y^{n-j}\right) V_{j}(b ; b)=0,0<u \leq b ; n \in \mathbb{Z}^{+} .
\end{aligned}
$$

Note that (2.4) together with the boundary conditions given by (2.1) determine $V_{n}(u ; b)$ for $0 \leq u \leq b$. Thus, for $u>b, V_{n}(u ; b)$ can be computed via (2.2).

From (2.4), we observe that the existence of the $n$-th moment of $D_{u, b}$ depends upon the existence of the $n$-th equilibrium distribution of the jump size random variable $Y$. This intuitively makes sense since in the dual model it is the jump which causes dividends to be paid and the size of the jumps determines the total dividend payout. This is in contrast to the classical model where the dividend rate is bounded by the premium rate $c$ and the $n$-th moment of the discounted dividends cannot exceed $(c / \delta)^{n}$. 


\subsection{Laplace Transform of the Time of Ruin}

With zero initial surplus, ruin occurs immediately and therefore

$$
\phi(0 ; b)=1 .
$$

In addition, when $u>b$, the surplus drops to level $b$ immediately due to the initial payment of dividends, and thus

$$
\phi(u ; b)=\phi(b ; b), u>b .
$$

For $0<u \leq b$, considering again a small increment $h>0$ leads to

$$
\begin{aligned}
\phi(u ; b)= & (1-\lambda h) e^{-\delta h} \phi(u-c h ; b)+\lambda h e^{-\delta h} \int_{0}^{b-(u-c h)} \phi(u-c h+y ; b) p(y) d y \\
& +\lambda h e^{-\delta h} \int_{b-(u-c h)}^{\infty} \phi(b ; b) p(y) d y+o(h) .
\end{aligned}
$$

In the same manner as (2.3) was obtained, we arrive at

$c \phi^{\prime}(u ; b)+(\lambda+\delta) \phi(u ; b)-\lambda \int_{0}^{b-u} \phi(u+y ; b) p(y) d y-\lambda \bar{P}(b-u) \phi(b ; b)=0$,

$0<u \leq b$.

The integro-differential equation (2.7), together with the boundary condition (2.5), determines $\phi(u ; b)$ for $0 \leq u \leq b$.

\section{Exact Solutions when the Jump Size Distribution is A COMBINATION OF EXPONENTIALS}

\subsection{Dividend Moments}

Assume the jump size random variable $Y$ is distributed as a combination of exponentials, so that its p.d.f. is given by

$$
p(y)=\sum_{i=1}^{r} A_{i} \beta_{i} e^{-\beta_{i} y}, y>0
$$

where $\beta_{i}>0$ for $i=1,2, \ldots, r$ and $\sum_{i=1}^{r} A_{i}=1$. Clearly, the $n$-th moment of $Y$ is given by

$$
E\left(Y^{n}\right)=n ! \sum_{i=1}^{r} \frac{A_{i}}{\beta_{i}^{n}}
$$


Furthermore, it follows by induction that

$$
\bar{P}_{n}(y)=\sum_{i=1}^{r} A_{i, n} e^{-\beta_{i} y}, y \geq 0 ; n \in \mathbb{N},
$$

where the $A_{i, n}$ 's can be computed recursively as

$$
A_{i, n}=\frac{A_{i, n-1} / \beta_{i}}{\sum_{j=1}^{r} A_{j, n-1} / \beta_{j}}, i=1,2, \ldots, r ; n \in \mathbb{Z}^{+},
$$

with starting values $A_{i, 0}=A_{i}$ for $i=1,2, \ldots, r$. With (3.1), (3.2), and (3.3), we can write (2.4) as

$$
\begin{aligned}
& c V_{n}^{\prime}(u ; b)+(\lambda+n \delta) V_{n}(u ; b)-\lambda \sum_{i=1}^{r} A_{i} \beta_{i} e^{\beta_{i} u} \int_{u}^{b} V_{n}(y ; b) e^{-\beta_{i} y} d y \\
& -\lambda \sum_{j=0}^{n}\left(\begin{array}{c}
n \\
j
\end{array}\right) \sum_{i=1}^{r} A_{i, n-j} e^{-\beta_{i}(b-u)}(n-j) ! \sum_{k=1}^{r} \frac{A_{k}}{\beta_{k}^{n-j}} V_{j}(b ; b)=0,0<u \leq b ; n \in \mathbb{Z}^{+},
\end{aligned}
$$

or equivalently,

$$
\begin{aligned}
& c V_{n}^{\prime}(u ; b)+(\lambda+n \delta) V_{n}(u ; b)-\lambda \sum_{i=1}^{r} A_{i} \beta_{i} e^{\beta_{i} u} \int_{u}^{b} V_{n}(y ; b) e^{-\beta_{i} y} d y \\
& -\lambda n ! \sum_{i=1}^{r}\left\{\sum_{j=0}^{n} \frac{A_{i, n-j}}{j !} \sum_{k=1}^{r} \frac{A_{k}}{\beta_{k}^{n-j}} V_{j}(b ; b)\right\} e^{\beta_{i}(u-b)}=0,0<u \leq b ; n \in \mathbb{Z}^{+} .
\end{aligned}
$$

We now follow the approach of Avanzi et al. (2007) and apply the operator

$$
\prod_{i=1}^{r}\left(\frac{d}{d u}-\beta_{i}\right)
$$

to (3.4), thereby resulting in $V_{n}(u ; b)$ satisfying an $(r+1)$-th order linear homogeneous differential equation (with constant coefficients which are independent of $u$ ). Therefore, we have that

$$
V_{n}(u ; b)=\sum_{k=0}^{r} C_{k, n} e^{\rho_{k, n} u}, 0 \leq u \leq b ; n \in \mathbb{Z}^{+},
$$

where the coefficients $C_{k, n}=C_{k, n}(b)$ and $\rho_{k, n}$ are to be determined.

Upon substitution of (3.5) into (3.4), we find, after some algebra, that 


$$
\begin{aligned}
\sum_{k=0}^{r} C_{k, n}\left(c \rho_{k, n}+\lambda+n \delta-\lambda \sum_{i=1}^{r} \frac{A_{i} \beta_{i}}{\beta_{i}-\rho_{k, n}}\right) e^{\rho_{k, n} u}+\lambda \sum_{i=1}^{r}\left(A_{i} \sum_{k=0}^{r} C_{k, n} \frac{\rho_{k, n}}{\beta_{i}-\rho_{k, n}} e^{\rho_{k, n} b}\right. \\
\left.-n ! \sum_{j=1}^{n-1} \frac{A_{i, n-j}}{j !} \sum_{k=1}^{r} \frac{A_{k}}{\beta_{k}^{n-j}} \sum_{l=0}^{r} C_{l, j} e^{\rho_{l, j} b}-n ! A_{i, n} \sum_{k=1}^{r} \frac{A_{k}}{\beta_{k}^{n}}\right) e^{\beta_{i}(u-b)}=0 \\
\quad 0<u \leq b ; n \in \mathbb{Z}^{+} .
\end{aligned}
$$

From the coefficients of $e^{\rho_{k, n} u}$ in (3.6), we have that

$$
c \rho_{k, n}+\lambda+n \delta-\lambda \sum_{i=1}^{r} \frac{A_{i} \beta_{i}}{\beta_{i}-\rho_{k, n}}=0, k=0,1, \ldots, r ; n \in \mathbb{Z}^{+} .
$$

In other words, for each fixed $n \in \mathbb{Z}^{+}$, the $\rho_{k, n}$ 's, $k=0,1, \ldots, r$, are the roots of Lundberg's fundamental equation (in $\xi$ ) under the dual model at force of interest $n \delta$, namely (e.g., see Avanzi et al. (2007, equation (4.9)))

$$
c \xi+\lambda+n \delta-\lambda \tilde{p}(-\xi)=0 .
$$

Similarly, looking at the coefficients of $e^{\beta_{i}(u-b)}$ in (3.6) leads to

$$
\begin{gathered}
A_{i} \sum_{k=0}^{r} C_{k, n} \frac{\rho_{k, n}}{\beta_{i}-\rho_{k, n}} e^{\rho_{k, n} b}=n ! \sum_{j=1}^{n-1} \frac{A_{i, n-j}}{j !} \sum_{k=1}^{r} \frac{A_{k}}{\beta_{k}^{n-j}} \sum_{l=0}^{r} C_{l, j} e^{\rho_{l, j} b}+n ! A_{i, n} \sum_{k=1}^{r} \frac{A_{k}}{\beta_{k}^{n}} \\
i=1,2, \ldots, r ; n \in \mathbb{Z}^{+} .
\end{gathered}
$$

Furthermore, it follows from (2.1) and (3.5) that

$$
\sum_{k=0}^{r} C_{k, n}=0, n \in \mathbb{Z}^{+}
$$

Therefore, for each fixed $n \in \mathbb{Z}^{+}$, (3.7) and (3.8) form a system of $r+1$ linear equations for the recursive (in terms of $n$ ) evaluation of the $C_{k, n}$ 's, $k=0,1, \ldots, r$. We remark that when $n=1$, this system is identical to that formed by equations (4.10) and (4.11) in Avanzi et al. (2007), and as such, becomes a starting point for the recursion.

\subsection{Laplace Transform of the Time of Ruin}

When the jump size distribution is governed by (3.1), one can easily follow the derivations in Section 3.1 to show that (2.7) gives rise to 


$$
\phi(u ; b)=\sum_{k=0}^{r} B_{k} e^{\rho_{k, 1} u}, 0 \leq u \leq b,
$$

where the $B_{k}$ 's satisfy

$$
\sum_{k=0}^{r} B_{k} \frac{\rho_{k, 1}}{\beta_{i}-\rho_{k, 1}} e^{\rho_{k, 1} b}=0, i=1,2, \ldots, r,
$$

and

$$
\sum_{k=0}^{r} B_{k}=1
$$

\section{Alternative Approach for Jump Size Distribution WITH RATIONAL LAPLACE TRANSFORM}

In this section, we assume that the jump size random variable $Y$ has a rational Laplace transform, i.e., we can write $\tilde{p}($.$) as$

$$
\tilde{p}(s)=\frac{l(s)}{h(s)},
$$

where $h($.$) and l($.$) are polynomials of degree m$ and (at most) $m-1$ respectively, with no common zeros. Furthermore, $h($.$) has no roots in the positive$ half plane, and $h(0)=l(0)$ ( since $\tilde{p}(0)=1)$. Without loss of generality, we assume the leading coefficient of $h($.$) is 1$. Then, the Laplace transform of the p.d.f. of the equilibrium random variable ${ }_{1} Y$ is given by

$$
\tilde{p}_{1}(s)=\frac{1-\tilde{p}(s)}{s E\left({ }_{0} Y\right)}=\frac{l_{1}(s)}{h(s)},
$$

where

$$
l_{1}(s)=\frac{h(s)-l(s)}{s E\left({ }_{0} Y\right)}
$$

is a polynomial of degree (at most) $m-1$ since $s=0$ is a root of $h(s)-l(s)$. Therefore, inductively, we write the Laplace transform of $p_{k}($.$) as$

$$
\tilde{p}_{k}(s)=\frac{l_{k}(s)}{h(s)}, k \in \mathbb{N},
$$

where the $l_{k}($.$) 's are given recursively by$

$$
l_{k}(s)=\frac{h(s)-l_{k-1}(s)}{s E\left({ }_{k-1} Y\right)}, k \in \mathbb{Z}^{+},
$$

with $l_{0}()=.l($.$) .$ 


\subsection{Dividend Moments}

Here, we follow the ideas in Section 6 of Avanzi et al. (2007). Letting $z=b-u$, we introduce the function

$$
W_{n}(z ; b)=V_{n}(b-z ; b)=V_{n}(u ; b), \quad 0 \leq z \leq b ; n \in \mathbb{N} .
$$

Note that

$$
W_{0}(z ; b)=1,0 \leq z \leq b,
$$

and

$$
W_{n}(b ; b)=V_{n}(0 ; b)=0, n \in \mathbb{Z}^{+} .
$$

With the above definition, (2.4) can be re-expressed as

$$
\begin{aligned}
& -c W_{n}^{\prime}(z ; b)+(\lambda+n \delta) W_{n}(z ; b)-\lambda \int_{0}^{z} W_{n}(z-y ; b) p(y) d y \\
& -\lambda \sum_{j=0}^{n}\left(\begin{array}{c}
n \\
j
\end{array}\right) \bar{P}_{n-j}(z) E\left(Y^{n-j}\right) W_{j}(0 ; b)=0,0 \leq z<b ; n \in \mathbb{Z}^{+},
\end{aligned}
$$

or equivalently,

$$
\begin{aligned}
& -c W_{n}^{\prime}(z ; b)+(\lambda+n \delta) W_{n}(z ; b)-\lambda \int_{0}^{z} W_{n}(z-y ; b) p(y) d y \\
& -\lambda \sum_{j=0}^{n}\left(\begin{array}{c}
n \\
j
\end{array}\right) p_{n-j+1}(z) E\left({ }_{n-j} Y\right) E\left(Y^{n-j}\right) W_{j}(0 ; b)=0,0 \leq z<b ; n \in \mathbb{Z}^{+} .
\end{aligned}
$$

As in Avanzi et al. (2007), we extend the domain for the definition of $W_{n}(z ; b)$ from $0 \leq z \leq b$ to $z \geq 0$, and denote the resulting function by $w_{n}(z)$. We then obtain the conditions

$$
w_{0}(z)=1, \quad z \geq 0,
$$

and

$$
w_{n}(b)=0, n \in \mathbb{Z}^{+} .
$$

Taking Laplace transforms on both sides of (4.1) with $w_{n}(z)$ in place of $W_{n}(z ; b)$ yields

$$
\begin{aligned}
& c w_{n}(0)-c s \tilde{w}_{n}(s)+(\lambda+n \delta) \tilde{w}_{n}(s)-\lambda \tilde{w}_{n}(s) \tilde{p}(s) \\
& \quad-\lambda \sum_{j=0}^{n}\left(\begin{array}{c}
n \\
j
\end{array}\right) \tilde{p}_{n-j+1}(s) E\left(_{n-j} Y\right) E\left(Y^{n-j}\right) w_{j}(0)=0, n \in \mathbb{Z}^{+} .
\end{aligned}
$$


Solving for $\tilde{w}_{n}(s)$ in the above equation, we obtain

$$
\begin{aligned}
\tilde{w}_{n}(s)= & \frac{\left\{1-\frac{\lambda}{c} E(Y) \tilde{p}_{1}(s)\right\} w_{n}(0)-\frac{\lambda}{c} \sum_{j=0}^{n-1}\left(\begin{array}{l}
n \\
j
\end{array}\right) \tilde{p}_{n-j+1}(s) E\left({ }_{n-j} Y\right) E\left(Y^{n-j}\right) w_{j}(0)}{s-\frac{\lambda+n \delta}{c}+\frac{\lambda}{c} \tilde{p}(s)}, \\
& n \in \mathbb{Z}^{+} .
\end{aligned}
$$

We remark that (4.3) holds true for an arbitrary jump size distribution. With the additional assumption of a rational Laplace transform, (4.3) then becomes

$$
\tilde{w}_{n}(s)=\frac{q_{n}(s)}{\left(s-\frac{\lambda+n \delta}{c}\right) h(s)+\frac{\lambda}{c} l(s)}, \quad n \in \mathbb{Z}^{+},
$$

where

$$
\begin{aligned}
q_{n}(s)= & \left\{h(s)-\frac{\lambda}{c} E(Y) l_{1}(s)\right\} w_{n}(0)-\frac{\lambda}{c} \sum_{j=0}^{n-1}\left(\begin{array}{c}
n \\
j
\end{array}\right) l_{n-j+1}(s) E\left({ }_{n-j} Y\right) E\left(Y^{n-j}\right) w_{j}(0), \\
& n \in \mathbb{Z}^{+} .
\end{aligned}
$$

We observe that the numerator of (4.4) is a polynomial of degree $m$ while the denominator is of degree $m+1$. As a result, we can apply a partial fraction decomposition to (4.4) and obtain

$$
\tilde{w}_{n}(s)=\sum_{k=0}^{m} \frac{Z_{k, n}}{s-R_{k, n}}, n \in \mathbb{Z}^{+},
$$

where for each fixed $n \in \mathbb{Z}^{+}$, the $R_{k, n}$ 's, $k=0,1, \ldots, m$, are the roots of Lundberg's fundamental equation (in $\xi$ ) under the classical risk model at force of interest $n \delta$, i.e.,

$$
c \xi-(\lambda+n \delta)+\lambda \tilde{p}(\xi)=0,
$$

and the $Z_{k, n}$ 's are given by

$$
Z_{k, n}=\frac{q_{n}\left(R_{k, n}\right)}{\prod_{i=0, i \neq k}^{m}\left(R_{k, n}-R_{i, n}\right)}, k=0,1, \ldots, m ; n \in \mathbb{Z}^{+} .
$$

Inversion of (4.6) immediately yields

$$
w_{n}(z)=\sum_{k=0}^{m} Z_{k, n} e^{R_{k, n} z}, \quad z \geq 0 ; n \in \mathbb{Z}^{+} .
$$


For each fixed $n \in \mathbb{Z}^{+}$, we note from (4.7) that each of the $Z_{k, n}$ 's involves the function $q_{n}($.$) , which in turn involves the constants w_{1}(0), w_{2}(0), \ldots, w_{n-1}(0)$. To determine these constants, we use condition (4.2) to get

$$
\sum_{k=0}^{m} Z_{k, n} e^{R_{k, n} b}=0, n \in \mathbb{Z}^{+},
$$

which, by application of (4.5) and (4.7), leads to

$$
\begin{gathered}
w_{n}(0)=\frac{\frac{\lambda}{c} \sum_{j=0}^{n-1}\left(\begin{array}{c}
n \\
j
\end{array}\right) E\left({ }_{n-j} Y\right) E\left(Y^{n-j}\right)\left\{\sum_{k=0}^{m} \frac{l_{n-j+1}\left(R_{k, n}\right)}{\prod_{i=0, i \neq k}^{m}\left(R_{k, n}-R_{i, n}\right)} e^{R_{k, n} b}\right\} w_{j}(0)}{\sum_{k=0}^{m} \frac{h\left(R_{k, n}\right)-\frac{\lambda}{c} E(Y) l_{1}\left(R_{k, n}\right)}{\prod_{i=0, i \neq k}^{m}\left(R_{k, n}-R_{i, n}\right)} e^{R_{k, n} b}}, \\
n \in \mathbb{Z}^{+} .
\end{gathered}
$$

We remark that the above equation enables the recursive computation of $w_{n}(0)$. Finally, the dividend moments are given by

$$
V_{n}(u ; b)=w_{n}(b-u)=\sum_{k=0}^{m} Z_{k, n} e^{R_{k, n}(b-u)}, 0 \leq u \leq b ; n \in \mathbb{Z}^{+} .
$$

\subsection{Laplace Transform of the Time of Ruin}

Following virtually the same set of procedures as in Section 4.1, we omit the tedious algebraic details and simply state that

$$
\phi(u ; b)=\varphi(b-u), \quad 0 \leq u \leq b,
$$

where

$$
\begin{gathered}
\varphi(z)=\sum_{k=0}^{m} L_{k} e^{R_{k, 1} z}, \quad z \geq 0, \\
L_{k}=\frac{\left\{h\left(R_{k, 1}\right)-\frac{\lambda}{c} E(Y) l_{1}\left(R_{k, 1}\right)\right\} \varphi(0)}{\prod_{i=0, i \neq k}^{m}\left(R_{k, 1}-R_{i, 1}\right)}, \quad k=0,1, \ldots, m,
\end{gathered}
$$

and

$$
\varphi(0)=\frac{1}{\sum_{k=0}^{m} \frac{h\left(R_{k, 1}\right)-\frac{\lambda}{c} E(Y) l_{1}\left(R_{k, 1}\right)}{\prod_{i=0, i \neq k}^{m}\left(R_{k, 1}-R_{i, 1}\right)} e^{R_{k, 1} b}} .
$$




\section{Discrete-Time Model}

In the discrete-time model, we assume, without loss of generality, that the company incurs an expense of 1 per unit time, and there is an upward jump of size $X_{k}$ at the end of the $k$-th time period, $k \in \mathbb{Z}^{+}$. We further assume that the jump sizes are i.i.d. having common distribution $X$ with mean $E(X)>1$. Let the probability mass function (p.m.f.) of $X$ be denoted by $g_{i}=\operatorname{Pr}(X=i), i \in \mathbb{N}$. Note that there is a probability $g_{0}$ of no upward jump at the end of each time period. Under the above description, the surplus process $\left\{U_{d}(k), k \in \mathbb{N}\right\}$ can be described by

$$
U_{d}(k)=u-k+\sum_{i=1}^{k} X_{i}
$$

where $u=U_{d}(0) \in \mathbb{N}$ is the initial surplus of the company. Ruin is said to occur if the surplus reaches level 0 .

Under a barrier strategy, a fixed dividend barrier $b \in \mathbb{Z}^{+}$is introduced to the above model. If $u>b$, a dividend of $u-b$ is payable immediately at time 0 . For $k \in \mathbb{Z}^{+}$, however, a dividend of $U_{d}(k-1)-1+j-b$ is payable at the end of the $k$-th time period if

(i) $U_{d}(k-1) \in \mathbb{Z}^{+}$, and

(ii) $X_{k}=j \geq b-U_{d}(k-1)+2$.

We assume no further dividends are payable beyond the time of ruin. We are then interested in the moments of the total discounted dividends under a force of interest $\alpha>0$ per unit time, and we denote the $n$-th moment by $V_{n, d}(u ; b)$. The 0 -th moment, $V_{0, d}(u ; b)$, is taken to be 1 for any $u$ and $b$. Mirroring the analysis in the continuous-time analogue, we denote the Laplace transform of the time of ruin (with argument $\alpha>0$ ) in the discrete-time model by $\phi_{d}(u ; b)$.

\subsection{Dividend Moments}

Analogous to (2.1) and (2.2), we have that

$$
V_{n, d}(0 ; b)=0, n \in \mathbb{Z}^{+},
$$

and

$$
V_{n, d}(u ; b)=\sum_{j=0}^{n}\left(\begin{array}{l}
n \\
j
\end{array}\right)(u-b)^{n-j} V_{j, d}(b ; b), u=b+1, b+2, \ldots ; n \in \mathbb{Z}^{+} .
$$

Then, by conditioning on $X_{1}$, we arrive at 


$$
\begin{aligned}
& V_{n, d}(u ; b)=e^{-n \alpha} \sum_{j=0}^{b-u+1} g_{j} V_{n, d}(u-1+j ; b) \\
& +e^{-n \alpha} \sum_{j=b-u+2}^{\infty} g_{j} \sum_{k=0}^{n}\left(\begin{array}{l}
n \\
k
\end{array}\right)(u-1+j-b)^{n-k} V_{k, d}(b ; b), u=1,2, \ldots, b ; n \in \mathbb{Z}^{+} .
\end{aligned}
$$

Therefore, for each fixed $n \in \mathbb{Z}^{+}, V_{n, d}(1 ; b), V_{n, d}(2 ; b), \ldots, V_{n, d}(b ; b)$ can be solved recursively from the above system of $b$ linear equations in terms of $V_{1, d}(b ; b)$, $V_{2, d}(b ; b), \ldots, V_{n-1, d}(b ; b)$. Then, for $u=b+1, b+2, \ldots, V_{n, d}(u ; b)$ can be computed via (5.2).

When the jump size random variable $X$ has infinite support, note that the system (5.3) involves infinite summation and direct application might pose some computational difficulties. However, it is possible to express (5.3) only in terms of finite summation when the moments of $X$ are known. Specifically, (5.3) can also be written as

$$
\begin{aligned}
& V_{n, d}(u ; b)=e^{-n \alpha} \sum_{j=0}^{b-u} g_{j} V_{n, d}(u-1+j ; b)+e^{-n \alpha}\left(1-\sum_{j=0}^{b-u} g_{j}\right) V_{n, d}(b ; b) \\
& +e^{-n \alpha} \sum_{k=0}^{n-1}\left(\begin{array}{l}
n \\
k
\end{array}\right) E\left[\left(\{X-(b-u+1)\}_{+}\right)^{n-k}\right] V_{k, d}(b ; b), u=1,2, \ldots, b ; n \in \mathbb{Z}^{+},
\end{aligned}
$$

where the stop-loss moments can be computed as

$$
\begin{aligned}
& E\left[\left(\{X-(b-u+1)\}_{+}\right)^{n}\right] \\
& =E\left[\{X-X \wedge(b-u+1)\}^{n}\right] \\
& =\sum_{k=0}^{n}\left(\begin{array}{l}
n \\
k
\end{array}\right)(-1)^{n-k} E\left[X^{k}\{X \wedge(b-u+1)\}^{n-k}\right] \\
& =\sum_{k=0}^{n}\left(\begin{array}{l}
n \\
k
\end{array}\right)(-1)^{n-k}\left\{\sum_{j=0}^{b-u+1} j^{n} g_{j}+\sum_{j=b-u+2}^{\infty}(b-u+1)^{n-k} j^{k} g_{j}\right\} \\
& =\sum_{k=0}^{n}\left(\begin{array}{l}
n \\
k
\end{array}\right)(-1)^{n-k}\left((b-u+1)^{n-k} E\left(X^{k}\right)-\sum_{j=0}^{b-u+1}\left\{(b-u+1)^{n-k} j^{k}-j^{n}\right\} g_{j}\right) \text {, } \\
& u=1,2, \ldots, b ; n \in \mathbb{Z}^{+} \text {. }
\end{aligned}
$$

Note that (5.5) only involves finite summation if the corresponding moments of $X$ are known. In particular, when $n=1,(5.5)$ reduces to

$$
\begin{aligned}
& E\left[\{X-(b-u+1)\}_{+}\right]=E(X)-(b-u+1)+\sum_{j=0}^{b-u+1}(b-u+1-j) g_{j}, \\
& u=1,2, \ldots, b .
\end{aligned}
$$


Returning to (5.4), we further distinguish between the case $b=1$ and $b>1$. For the case $b=1$, with the use of $(5.1),(5.4)$ becomes

$$
V_{n, d}(1 ; 1)=e^{-n \alpha}\left(1-g_{0}\right) V_{n, d}(1 ; 1)+e^{-n \alpha} \sum_{k=0}^{n-1}\left(\begin{array}{l}
n \\
k
\end{array}\right) E\left[\left\{(X-1)_{+}\right\}^{n-k}\right] V_{k, d}(1 ; 1), n \in \mathbb{Z}^{+} .
$$

Solving for $V_{n, d}(1 ; 1)$ yields

$$
V_{n, d}(1 ; 1)=\frac{e^{-n \alpha}}{1-e^{-n \alpha}\left(1-g_{0}\right)} \sum_{k=0}^{n-1}\left(\begin{array}{l}
n \\
k
\end{array}\right) E\left[\left\{(X-1)_{+}\right\}^{n-k}\right] V_{k, d}(1 ; 1), n \in \mathbb{Z}^{+} .
$$

For $n=1$, application of (5.6) to (5.7) leads to

$$
V_{1, d}(1 ; 1)=\frac{e^{-\alpha}\left\{E(X)-1+g_{0}\right\}}{1-e^{-\alpha}\left(1-g_{0}\right)}=1+\frac{e^{-\alpha} E(X)-1}{1-e^{-\alpha}\left(1-g_{0}\right)} .
$$

We note that (5.8) has the following interesting implication, namely, the sign of $V_{1, d}(1 ; 1)-1$ is identical to that of $e^{-\alpha} E(X)-1$. In other words, $V_{1, d}(1 ; 1)$ is greater (less) than 1 if and only if $E(X)$ is greater (less) than $e^{\alpha}$.

We now turn our attention to the case where $b>1$. In such instances, a system of linear equations has to be solved and as such, results are stated in terms of matrices. The system of linear equations (5.4) can be expressed in matrix form as

$$
\mathbf{A}_{n}(b) \mathbf{V}_{n, d}(b)=\Pi_{n}(b), \quad b>1 ; n \in \mathbb{Z}^{+},
$$

where the involved quantities are defined as

$$
\begin{gathered}
\mathbf{A}_{n}(b)=e^{-n \alpha} \mathbf{Q}-\mathbf{I}_{b}, \\
\mathbf{Q}=\left(\begin{array}{cccccc}
g_{1} & g_{2} & g_{3} & \cdots & g_{b-1} & 1-\sum_{j=0}^{b-1} g_{j} \\
g_{0} & g_{1} & g_{2} & \cdots & g_{b-2} & 1-\sum_{j=0}^{b-2} g_{j} \\
0 & g_{0} & g_{1} & \cdots & g_{b-3} & 1-\sum_{j=0}^{b-3} g_{j} \\
\vdots & \vdots & \vdots & \ddots & \vdots & \vdots \\
0 & 0 & 0 & \cdots & g_{1} & 1-g_{0}-g_{1} \\
0 & 0 & 0 & \cdots & g_{0} & 1-g_{0}
\end{array}\right), \\
\mathbf{V}_{n, d}(b)=\left(V_{n, d}(1 ; b), V_{n, d}(2 ; b), \ldots, V_{n, d}(b ; b)\right)^{T}, \\
\pi_{n}(u ; b)=-e^{-n \alpha} \sum_{k=0}^{n-1}\left(\begin{array}{l}
n \\
k
\end{array}\right) E\left[\left(\{X-(b-u+1)\}_{+}\right)^{n-k}\right] V_{k, d}(b ; b), u=1,2, \ldots, b,
\end{gathered}
$$


and $\mathbf{I}_{b}$ is a $b \times b$ identity matrix. Note that the stop-loss moments appearing in $\pi_{n}(u ; b)$ can be computed using (5.5). Then, from (5.9), $\mathbf{V}_{n, d}(b)$ is given by

$$
\mathbf{V}_{n, d}(b)=\left[\mathbf{A}_{n}(b)\right]^{-1} \boldsymbol{\Pi}_{n}(b), b>1 ; n \in \mathbb{Z}^{+} .
$$

We remark that the above inverse $\left[\mathbf{A}_{n}(b)\right]^{-1}$ always exists. To see this, we can imagine $e^{-n \alpha} \mathbf{Q}$ to be the transition probability matrix among the transient states of a discrete-time Markov chain, and therefore $\left(\mathbf{I}_{b}-e^{-n \alpha} \mathbf{Q}\right)^{-1}$ is always known to exist.

\subsection{Laplace Transform of the Time of Ruin}

Identical to (2.5) and (2.6), we have

$$
\phi_{d}(0 ; b)=1
$$

and

$$
\phi_{d}(u ; b)=\phi_{d}(b ; b), u=b+1, b+2, \ldots
$$

Conditioning on $X_{1}$, we obtain

$\phi_{d}(u ; b)=e^{-\alpha} \sum_{j=0}^{b-u} g_{j} \phi_{d}(u-1+j ; b)+e^{-\alpha}\left(1-\sum_{j=0}^{b-u} g_{j}\right) \phi_{d}(b ; b), u=1,2, \ldots, b$.

We again distinguish between the cases $b=1$ and $b>1$. For $b=1$, with the application of $(5.10),(5.11)$ reduces to

$$
\phi_{d}(1 ; 1)=e^{-\alpha} g_{0}+e^{-\alpha}\left(1-g_{0}\right) \phi_{d}(1 ; 1),
$$

which immediately yields

$$
\phi_{d}(1 ; 1)=\frac{e^{-\alpha} g_{0}}{1-e^{-\alpha}\left(1-g_{0}\right)} .
$$

Note that (5.12) represents the Laplace transform of a zero-truncated geometric random variable. This makes sense because when $u=b=1$, the time of ruin is $k, k \in \mathbb{Z}^{+}$, if there is a positive jump in each of the first $k-1$ periods and no jump at the end of the $k$-th period.

Regarding the case $b>1$, similar to (5.9), (5.11) can be expressed as

$$
\mathbf{A}_{1}(b) \boldsymbol{\Phi}_{d}(b)=\mathbf{Y}, \quad b>1,
$$

where

$$
\boldsymbol{\Phi}_{d}(b)=\left(\phi_{d}(1 ; b), \phi_{d}(2 ; b), \ldots, \phi_{d}(b ; b)\right)^{T}
$$


and

$$
\mathbf{Y}=\left(-g_{0} e^{-\alpha}, 0, \ldots, 0\right)^{T}
$$

Clearly, (5.13) implies

$$
\boldsymbol{\Phi}_{d}(b)=\left[\mathbf{A}_{1}(b)\right]^{-1} \mathbf{Y}, b>1 .
$$

\section{Approximation of the Continuous-Time Model}

In this section, we follow the essential ideas in Dickson and Waters (1991, 2004) to establish a connection between the continuous-time model and the discrete-time model in Section 5. In other words, the quantities $V_{n}(u ; b)$ and $\phi(u ; b)$ in the continuous-time model can be approximated by the corresponding quantities in the discrete-time model when exact solutions are not available.

Without loss of generality, we assume, in the continuous-time model, that the jump size random variable $Y$ has mean $E(Y)=1$, and the Poisson rate of jump arrival is $\lambda=1$. Therefore, the rate of expenses per unit time is $c=1 /(1+\theta)$. Our goal is to approximate $V_{n}(u ; b)$ and $\phi(u ; b)$ under a force of interest $\delta>0$. Similar to the steps outlined in Dickson (2005, pp. 147-148), our approximation procedure involves three steps.

\section{Step 1: Discretization of $Y$}

Discretize the random variable $Y$ on $\{0,1 / \beta, 2 / \beta, \ldots\}$ for some chosen parameter $\beta>0$, which is referred to as the scaling factor. If we call the discretized random variable ${ }_{(1)} Y$, the discretization procedure should be such that ${ }_{(1)} Y$ approximates $Y$. The mean preserving method (e.g., see Dickson (2005, p. 80), or De Vylder and Goovaerts (1988, Section 7) for further details) is used so that the mean of ${ }_{(1)} Y$ is also 1 . Denoting the $n$-th moment of the total discounted dividends by ${ }_{1} V_{n}(u ; b)$ and the Laplace transform of the time of ruin by ${ }_{1} \phi(u ; b)$ in the same compound Poisson model but with jump size random variable $_{(1)} Y$, ideally we have

$$
V_{n}(u ; b) \simeq{ }_{1} V_{n}(u ; b), n \in \mathbb{Z}^{+},
$$

and

$$
\phi(u ; b) \simeq{ }_{1} \phi(u ; b)
$$

if ${ }_{(1)} Y$ is a good approximation of $Y$.

\section{Step 2: Change of monetary unit}

Define the random variable ${ }_{(2)} Y=\beta \cdot{ }_{(1)} Y$ with mean $\beta$. Note that ${ }_{(2)} Y$ is distributed on $\mathbb{N}$. If ${ }_{2} V_{n}(u ; b)$ denotes the $n$-th moment of the total discounted dividends and ${ }_{2} \phi(u ; b)$ denotes the Laplace transform of the time of ruin in the compound Poisson model with jump size random variable ${ }_{(2)} Y$, jump rate 1, 
force of interest $\delta$, constant rate of expenses per unit time $\beta /(1+\theta)$, initial surplus $u$, and barrier $b$, it is immediate that

$$
{ }_{1} V_{n}(u ; b)=\frac{1}{\beta^{n}}{ }_{2} V_{n}(\beta u ; \beta b), n \in \mathbb{Z}^{+},
$$

and

$$
{ }_{1} \phi(u ; b)={ }_{2} \phi(\beta u ; \beta b) .
$$

Then, from (6.1) and (6.2), ideally we have

$$
V_{n}(u ; b) \simeq \frac{1}{\beta^{n}}{ }_{2} V_{n}(\beta u ; \beta b), n \in \mathbb{Z}^{+},
$$

and

$$
\phi(u ; b) \simeq{ }_{2} \phi(\beta u ; \beta b) .
$$

\section{Step 3: Change of time unit}

If we change the time unit of the model in Step 2 such that the rate of expenses per unit time is 1 , then the model in Step 2 is equivalent to a model where the force of interest per unit time is $\delta(1+\theta) / \beta$, and the jump size random variable per unit time, defined as ${ }_{(3)} Y$, has a compound Poisson distribution with rate $(1+\theta) / \beta$ and secondary distribution ${ }_{(2)} Y$. This is precisely the model described in Section 5 where $\alpha=\delta(1+\theta) / \beta$ and the jump size random variable $X$ has the same distribution as ${ }_{(3)} Y$. Therefore, we arrive at the approximation

$$
V_{n}(u ; b) \simeq \frac{1}{\beta^{n}} V_{n, d}(\beta u ; \beta b), n \in \mathbb{Z}^{+},
$$

and

$$
\phi(u ; b) \simeq \phi_{d}(\beta u ; \beta b) .
$$

We remark that in order to evaluate the p.m.f. of ${ }_{(3)} Y$, the well-known $(a, b, 0)$ Panjer recursion can be used (e.g., see Klugman et al. (2004, pp. 91-92)). Moreover, we expect the approximation to improve as the value of $\beta$ increases.

\section{Optimal Dividends: Gerber-Lin-Yang Modification}

Avanzi et al. (2007) consider the problem of maximizing the expected value of total discounted dividends in the dual model. In this section, we consider a modified problem regarding the choice of the optimal dividend barrier.

Our modified problem is inspired by Dickson and Waters (2004) as well as Gerber et al. (2006a). Both papers consider dividend problems in the classical risk model. Dickson and Waters (2004) argue that the shareholders should be responsible for the deficit at ruin, and therefore one should maximize the 
difference between the expected discounted dividends and the discounted deficit at ruin. This is referred to as the 'Dickson-Waters modification' by Gerber et al. (2006b). In the dual model, however, this is no longer applicable since the deficit at ruin always equals 0 . Gerber et al. (2006a) consider a more general problem of maximizing the difference between the expected discounted dividends and the expected discounted penalty at ruin. Therefore, if we assume that a constant penalty of $w \geq 0$ should be paid by the company at the time of ruin, then we aim at maximizing (with respect to $b$ ) the quantity

$$
\gamma(u ; b, w)=V_{1}(u ; b)-w \phi(u ; b) .
$$

We refer to this maximization problem as the 'Gerber-Lin-Yang' modification. Note that the value $w$ can be interpreted as a 'weight' assigned to the Laplace transform of the time of ruin. This means that when $w$ is small, we are more concerned about the expected amount of total discounted dividends paid, whereas if $w$ is large, we are more concerned with the possibility of early ruin.

Our studies reveal that given a fixed value of $w \geq 0$, the function $\gamma(u ; b, w)$ is maximized at $b=b_{w}^{*}$ for any value of $u \geq 0$. In other words, the optimal barrier is independent of the initial surplus $u$ but is dependent on the value of the penalty $w$ at ruin. In the case where $w=0$, Avanzi et al. (2007) argue that, in general, the optimal barrier $b_{0}^{*}$ is positive. Indeed, if we follow their same line of logic, it is possible to show that $b_{w}^{*}$ is positive for any fixed $w \geq 0$. From (2.1), (2.3), (2.5), and (2.7), it is obvious that (7.1) satisfies the integro-differential equation

$$
\begin{aligned}
& c \gamma^{\prime}(u ; b, w)+(\lambda+\delta) \gamma(u ; b, w)-\lambda \int_{0}^{b-u} \gamma(u+y ; b, w) p(y) d y \\
& \quad-\lambda \int_{b-u}^{\infty}(u-b+y) p(y) d y-\lambda \gamma(b ; b, w) \bar{P}(b-u)=0, \quad 0<u \leq b,
\end{aligned}
$$

with boundary condition

$$
\gamma(0 ; b, w)=-w .
$$

If we now let $u$ and $b$ both go to 0 in (7.2), use (7.3), and then solve for $\gamma^{\prime}(0 ; 0, w)$, we obtain

$$
\gamma^{\prime}(0 ; 0, w)=\frac{\lambda}{c} \int_{0}^{\infty} y p(y) d y+\frac{\delta}{c} w=1+\theta+\frac{\delta}{c} w>1
$$

since $\theta>0$ and $w \geq 0$. By continuity, we argue that for each fixed $w \geq 0$ there exists $b_{w}>0$ such that

$$
\gamma^{\prime}\left(u ; b_{w}, w\right)>1, \quad 0<u<b_{w} .
$$


Replacing $u$ by $y$ in the above condition and integrating (with respect to $y$ ) from 0 to $u$, we obtain, by again applying (7.3), that for each fixed $w \geq 0$ there exists $b_{w}>0$ such that

$$
\gamma\left(u ; b_{w}, w\right)>u-w, \quad 0<u<b_{w} .
$$

Since $\gamma(u ; 0, w)=u-w$ from (7.1), it is always more optimal to choose such a barrier of $b_{w}$ than to choose a barrier of 0 . Therefore, in general, the optimal barrier $b_{w}^{*}$ is positive.

Avanzi et al. (2007, equation (5.9)) derive an interesting equation regarding the optimal barrier in the case when $w=0$. Expressed in our notation, it is given by

$$
V_{1}\left(b_{0}^{*} ; b_{0}^{*}\right)=\frac{\mu}{\delta},
$$

which happens to be the present value of a perpetuity payable at rate $\mu$. In fact, this result can be extended to

$$
\gamma\left(b_{w}^{*} ; b_{w}^{*}, w\right)=\frac{\mu}{\delta},
$$

for any $w \geq 0$. We omit the proof of this result as it is identical to that of Avanzi et al. (2007).

\section{Numerical ILluStrations}

This section is devoted to some numerical examples which illustrate the application of our results from the previous sections. In all examples, we assume the Poisson jump rate $\lambda$ to be 1 . We begin by considering the following four jump size distributions which all have rational Laplace transform:

Example 1: Damped squared sine distribution

$$
p(y)=8 e^{-2 y} \sin ^{2} y, \quad y>0 .
$$

Example 2: Mixture of an exponential and two Erlangs

$$
p(y)=\frac{1}{2}\left(2^{2} y e^{-2 y}\right)+\frac{1}{8}\left(2.5 e^{-2.5 y}\right)+\frac{3}{8}\left(\frac{2.5^{3} y^{2} e^{-2.5 y}}{2 !}\right), y>0 .
$$

Example 3: Damped sine distribution

$$
p(y)=2 e^{-y}(1-\sin y), y>0 .
$$

Example 4: Mixture of two Erlangs

$$
p(y)=\frac{1}{4}\left(0.6^{2} y e^{-0.6 y}\right)+\frac{3}{4}\left(9^{2} y e^{-9 y}\right), \quad y>0 .
$$




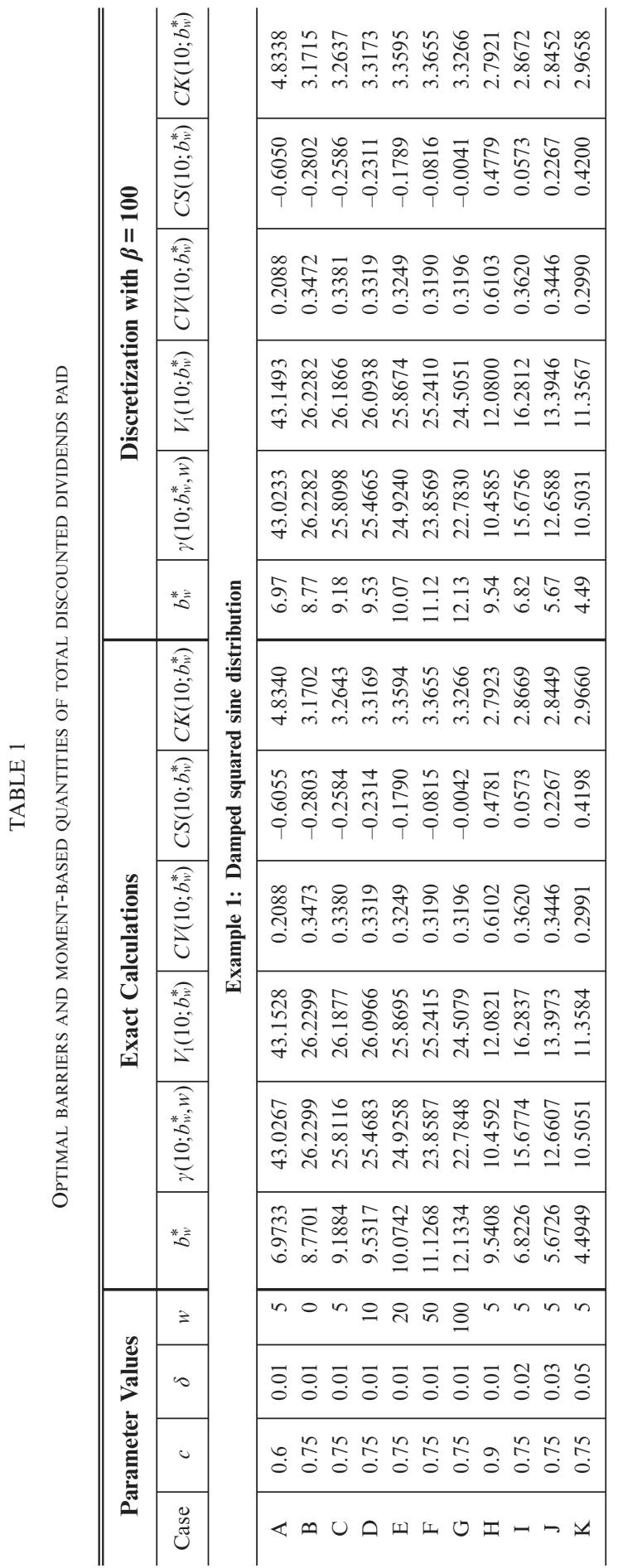

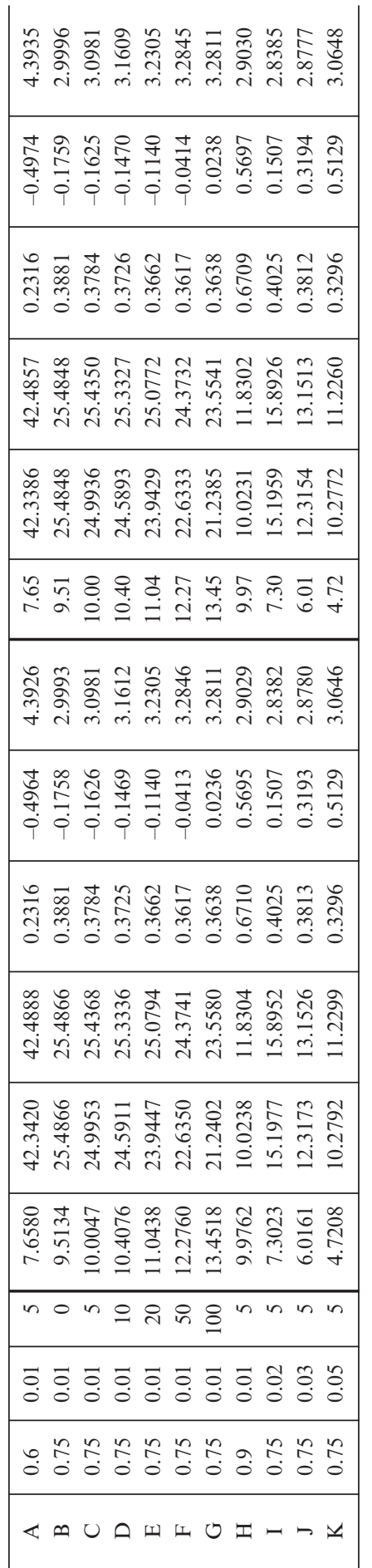




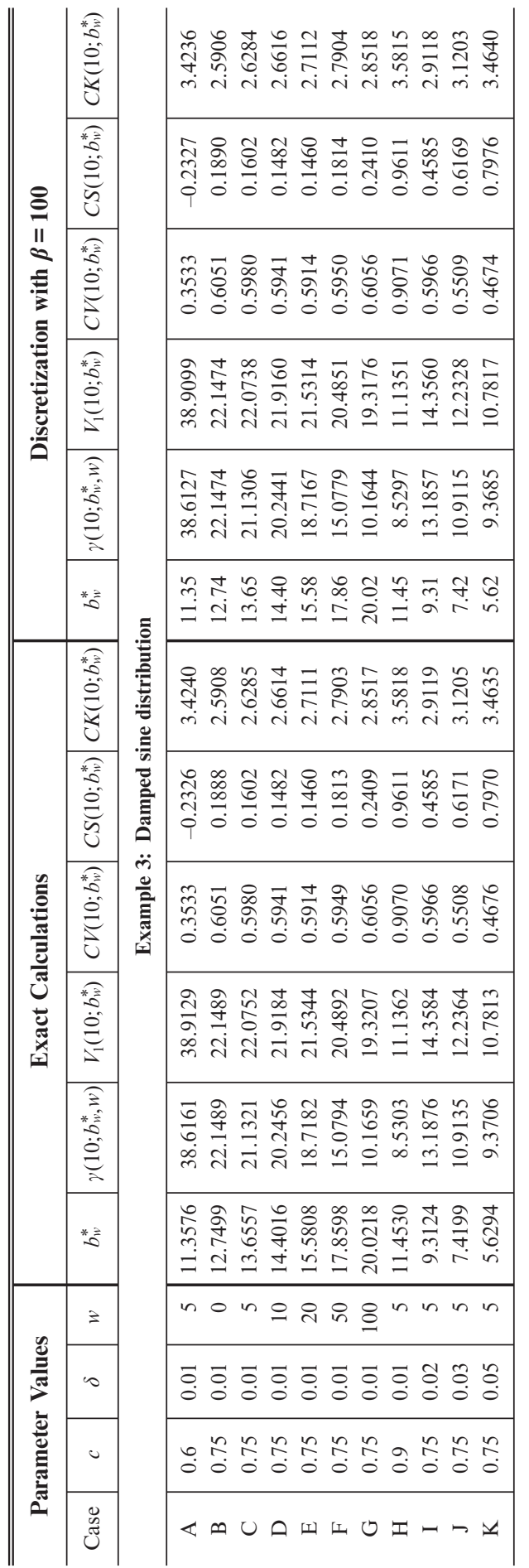

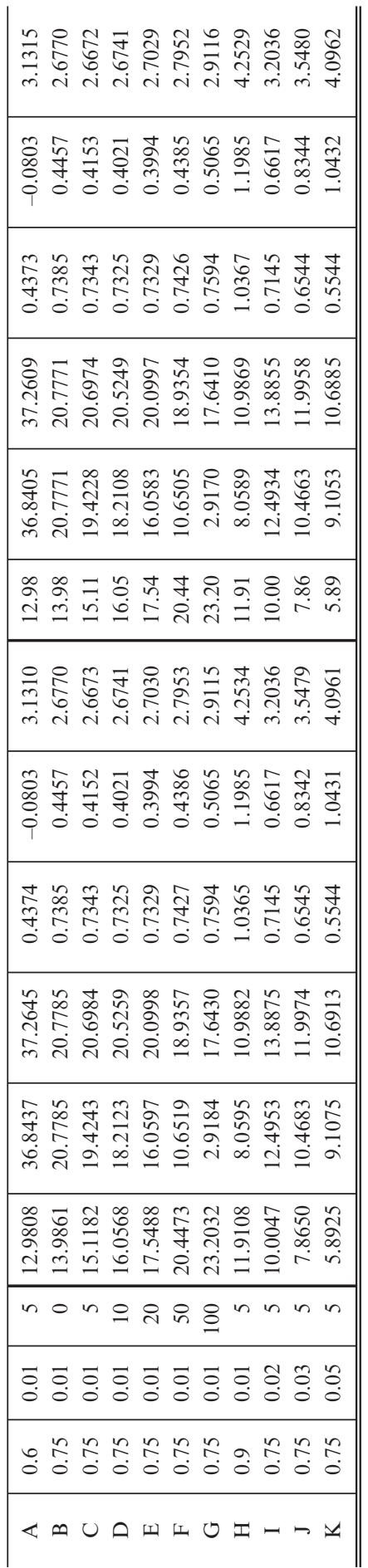


While the above four distributions all have mean 1, they possess different amounts of variability. Specifically, the coefficients of variation corresponding to the jump size distributions in Examples 1-4 are (rounded to two decimal places) $0.50,0.71,1.41$, and 1.80 respectively.

For each of the four examples above, Table 1 displays both the exact and approximated (via discretization with a scaling factor of $\beta=100$ ) values of $b_{w}^{*}$, $\gamma\left(10 ; b_{w}^{*}, w\right)$, and $V_{1}\left(10 ; b_{w}^{*}\right)$ obtained for various combinations of the parameters $c, \delta$, and $w$. In addition, since we have explicit formulae for the higher moments of $D_{u, b_{w}^{*}}$, Table 1 also presents the coefficient of variation, the coefficient of skewness, and the coefficient of kurtosis of $D_{u, b_{w}^{*}}$ for $u=10$. These three moment-based quantities are denoted by $C V\left(10 ; b_{w}^{*}\right), C S\left(10 ; b_{w}^{*}\right)$, and $C K\left(10 ; b_{w}^{*}\right)$ respectively.

We remark that with $\beta$ set at 100 , all values of $b_{w}^{*}$ on the discretized side of Table 1 are specified to only two decimal places of accuracy. Nevertheless, we observe that the results from the approximation are very close to those obtained via the exact method. We also remark that in cases $\mathrm{A}, \mathrm{C}$, and $\mathrm{H}$ where only $c$ is varied, there seems to be no monotonic relationship between $b_{w}^{*}$ and $c$. In particular, $b_{w}^{*}$ appears to first increase and then decrease with $c$. On the other hand, in cases B, C, D, E, F, and G, the value of $w$ is only varied and, as expected, the value of $b_{w}^{*}$ increases with $w$ in each example. This intuitively makes sense since a larger value of $w$ warrants a greater concern of earlier ruin, and so $b_{w}^{*}$ has to increase for safety purposes. We also observe that in cases $\mathrm{C}, \mathrm{I}, \mathrm{J}$, and $\mathrm{K}$ where only $\delta$ is varied, $b_{w}^{*}$ decreases as $\delta$ increases in all examples.

For all cases of Table 1, the value of $b_{w}^{*}$ appears to have the same ordering as the coefficient of variation or variance (since we are fixing the mean to be 1) of the jump size distribution. This comment is in fact made in Avanzi et al. (2007) for the case $w=0$. However, this appears to be true only when we are making the comparison among light-tailed distributions. Specifically, let us now consider the situation where jump sizes follow a lognormal distribution with p.d.f.

$$
p(y)=\frac{1}{y(9 / 7) \sqrt{2 \pi}} \exp \left\{-\frac{1}{2}\left(\frac{\ln y+81 / 98}{9 / 7}\right)^{2}\right\}, y>0 .
$$

This distribution has a mean of 1 , but its coefficient of variation is 2.05 and is higher than all the distributions in Examples 1-4. Applying a discretization with $\beta=100$, two graphs are plotted assuming $c=0.75$ and $\delta=0.01$. Figure 1 shows a plot of $\gamma(u ; b, 5)$ against $b$ for various fixed values of $u$. From Figure 1 , it appears that the optimal value of $b$ which maximizes $\gamma(u ; b, 5)$ is independent of $u$, and we can indeed confirm that for each value of $u$ in Figure 1, the optimal value of $b$ to two decimal places is 13.93 . This is significantly lower than 15.12, which is the corresponding optimal value in Example 4 where the jump size distribution has a smaller coefficient of variation of 1.80. Therefore, it would seem that a heavy-tailed distribution such as the lognormal exhibits 


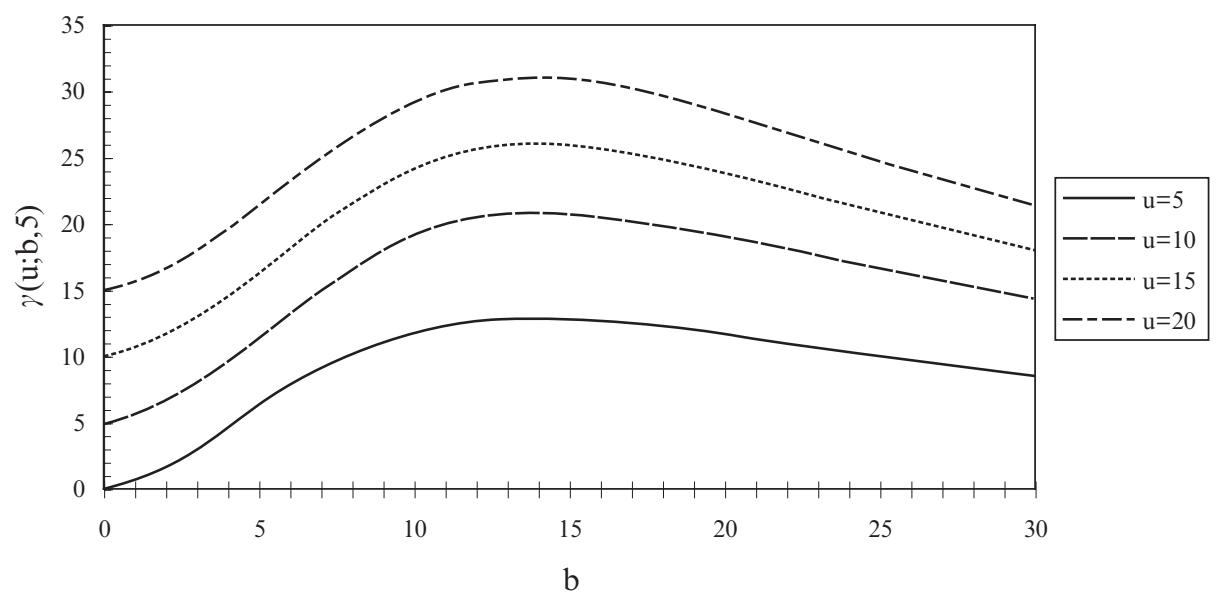

FIGURE 1: Plot of $\gamma(u ; b, 5)$ against $b$ for various fixed values of $u$.

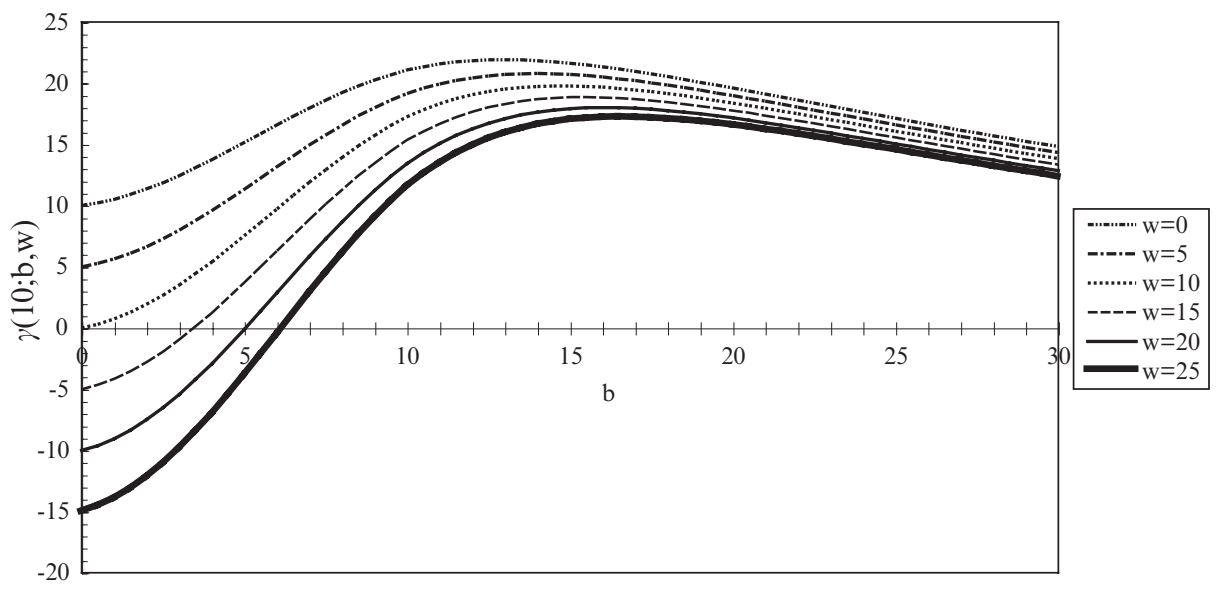

Figure 2: Plot of $\gamma(10 ; b, w)$ against $b$ for various fixed values of $w$.

a different sort of behaviour. To add further credence to our claim, we also performed the same discretization procedure assuming a Pareto jump size distribution with a mean of 1 and coefficient of variation of 1.41 , identical to the one in Example 3. We found the value of $b_{5}^{*}$ to be 12.41, again significantly lower than 13.66, the corresponding value of $b_{5}^{*}$ in Example 3. Thus, in general, we cannot say that $b_{w}^{*}$ increases with the variance of the jump size distribution.

Finally, Figure 2 depicts the behaviour of $\gamma(10 ; b, w)$ as a function of $b$ for various fixed values of $w$ in the lognormal case. Note that $b_{w}^{*}$ increases with $w$, and this is consistent with our findings in Table 1. 


\section{ACKNOWLEDGEMENTS}

The authors would like to thank the referees for their useful remarks and suggestions which helped to improve this paper. The authors would also like to thank Professors Harry Panjer and Gordon Willmot for their helpful comments and use of references in the literature. This work has been assisted by the Natural Sciences and Engineering Research Council of Canada.

\section{REFERENCES}

Avanzi, B., Gerber, H.U. and Shiu, E.S.W. (2007) Optimal dividends in the dual model. Insurance: Mathematics and Economics, 41, 111-123.

De VyLDER, F. and GoOvaerTs, M.J. (1988) Recursive calculation of finite-time ruin probabilities. Insurance: Mathematics and Economics, 7, 1-7.

Dickson, D.C.M. (2005) Insurance Risk and Ruin, Cambridge University Press, Cambridge.

Dickson, D.C.M. and WATERS, H.R. (1991) Recursive calculation of survival probabilities. ASTIN Bulletin, 21, 199-221.

Dickson, D.C.M. and WATERS, H.R. (2004) Some optimal dividends problems. ASTIN Bulletin, 34, 49-74.

Gerber, H.U., Lin, X.S. and YANG, H. (2006a) A note on the dividends-penalty identity and the optimal dividend barrier. ASTIN Bulletin, 36, 489-503.

Gerber, H.U., Shiu, E.S.W. and Smith, N. (2006b) Maximizing dividends without bankruptcy. ASTIN Bulletin, 36, 5-23.

Klugman, S.A., Panjer, H.H. and Willmot, G.E. (2004) Loss Models: From Data to Decisions, 2nd edition, Wiley, New York.

MazzA, C. and Rullière, D. (2004) A link between wave governed random motions and ruin processes. Insurance: Mathematics and Economics, 35, 205-222.

SEal, H.L. (1969) Stochastic Theory of a Risk Business, Wiley, New York.

Willmot, G.E., Drekic, S. and CAI, J. (2005) Equilibrium compound distributions and stoploss moments. Scandinavian Actuarial Journal, 6-24.

\section{ERIC C.K. CheUNG}

Department of Statistics and Actuarial Science

University of Waterloo

200 University Avenue West

Waterloo, Ontario, Canada N2L $3 G 1$

STEVE DReKIC (corresponding author)

Department of Statistics and Actuarial Science

University of Waterloo

200 University Avenue West

Waterloo, Ontario, Canada N2L $3 G 1$

E-mail:sdrekic@math.uwaterloo.ca 